\title{
Distributed MPC with Time-varying Communication Network: A Density-dependent Population Games Approach
}

\author{
J. Barreiro-Gomez, N. Quijano, Senior Member, IEEE, and C. Ocampo-Martinez, Senior Member, IEEE
}

\begin{abstract}
This work addresses distributed control design by using density-dependent population dynamics. Furthermore, stability of the equilibrium point under this proposed class of population dynamics is studied, and the relationship between the equilibrium point of density-dependent population games (DDPG) and the solution of constrained optimization problems is shown. Finally, a distributed predictive control is designed with the proposed density-dependent dynamics, and contemplating a time-varying communication network.

Index Terms - Density games, population dynamics, revision protocol, distributed model predictive control, time-varying communication network.
\end{abstract}

\section{INTRODUCTION}

One of the main properties of the population dynamics approach is that the proportion of agents, under these type of dynamics, evolve inside an invariant set. In this regard, population dynamics are able to satisfy a unique coupled constraint. Besides, dynamics evolve towards a maximum point of a concave function under a specific type of games known as full-potential games. Moreover, the mentioned features are preserved in a non-centralized manner by using the distributed version of the population dynamics, which have the same properties as their classical counterpart, i.e., invariance of the simplex set and asymptotic convergence to the equilibrium point [1]. On the other hand, large-scale systems become more challenging to control as their number of states and manipulated variables increase. Furthermore, graph theory has been used to describe the possible interaction or possible information sharing among local decision makers operating to control a system in a distributed way. Therefore, distributed population dynamics have become a powerful tool in the design of engineering control systems under distributed topologies and/or communication system structures.

The properties of the distributed population dynamics have been exploited to solve resource allocation problems under non-centralized communication structures. Nevertheless, the necessity to include more constraints, different from the one

J. Barreiro-Gomez and N. Quijano are with Departamento de Ingeniería Eléctrica y Electrónica, Universidad de los Andes, Carrera $1^{\mathrm{A}}$ No $18 \mathrm{~A}-10$, Colombia $\{j$.barreiro135, nquijano\}@uniandes.edu.co.

J. Barreiro-Gomez and C. Ocampo-Martinez are with the Automatic Control Department, Universitat Politècnica de Catalunya, Institut de Robòtica i Informàtica Industrial (CSIC-UPC), Llorens i Artigas, 4-6, 08028 Barcelona, Spain. \{jbarreiro, cocampo\}@iri.upc.edu

Authors thank COLCIENCIAS (grant 6172) and AGAUR Ref. FI-2014 for supporting J. Barreiro-Gomez. Authors thank the projects "Drenaje urbano y cambio climático: hacia los sistemas de alcantarillado del futuro. Fase II", Mexichem, Colombia, and ECOCIS (Ref. DPI2013-48243-C2-1-R) from the Spanish Ministry of Science and Education, for partially supporting this research. associated to the simplex set, has gotten special importance to be addressed with population games. For instance in [2], a population dynamics approach, able to solve optimization problems considering multiple constraints, is presented. The authors propose to divide the optimization problems into several smaller sub-problems, and dynamical feasible regions are considered in order to achieve an agreement that solves the non-divided optimization problem, allowing also the population size vary along the time.

From a biological perspective, allowing variations of the population size illustrates a special situation in which death and birth, or reproduction rates, are considered as in [3][5]. Density games have been studied to model a population with reproductive rates. However, these dynamics have not been deduced from a version of the general dynamics known as mean dynamics, neither imposing different rules on revision protocols. The reason is that the density-dependent dynamics have been mainly studied in a different context (i.e., biology sciences [3][5]) from the context where the mean dynamics have been mainly applied (e.g., economics [8] and optimization-based control engineering [1]). Besides, equilibrium points in this type of dynamics have not been related to the solution of constrained optimization problems. Regarding control design, in [7] the dynamics with density dependence presented in [3] have been used for control purposes. Nonetheless, distributed density-dependent population dynamics have not been introduced as a potential tool for control design. Then, the aim of this paper is to propose a general methodology for the deduction of new distributed density-dependent population dynamics and discussing their role in optimization-based control design. Briefly, this paper presents a density-dependent game approach that, as in [2], allows the population size vary. However, the proposed density-dependent game approach relaxes the conditions over the graph topology (associated to cliques) presented in [2]. Indeed, the design of distributed densitydependent population-dynamics-based MPC controller under time-varying communication network topology is addressed, becoming an alternative methodology in the design of distributed MPC controllers [4].

Therefore, the contribution of this paper is twofold. First, it is proposed to extend the mean dynamics with strategyconstrained interactions by considering a reproduction rate parameter. Then, by using the pairwise proportional imitation protocol (protocol that generates the replicator dynamics from the mean dynamics [8]), the distributed densitydependent replicator dynamics are deduced. Afterwards, it is shown that these density dynamics may be used to solve 
distributed constrained optimization problems when selecting properly the fitness functions in the strategic interaction based on the Lagrangian of a cost function. As a second contribution, and taking advantage of the properties that densitydependent population games (DDPG) have, a distributed model predictive control (MPC) design is proposed based on the distributed density-dependent replicator dynamics. Besides, the relationship between the strategy-interaction structure and the distributed communication network for the distributed MPC is discussed. Then, it is shown that the strategy-interaction structure can be modified dynamically along the time by adding conditions over the optimization problem constraints depending on the current system state, leading this fact to a time-varying communication network for control purposes.

The remainder of this paper is organized as follows. Section II presents the preliminaries of population games and the density-dependent approach. Section III introduces the density-dependent replicator dynamics and the stability of their equilibrium point is formally proven. Afterwards, Section IV shows the design of a distributed density-dependent population-dynamics-based MPC. The presented approach is implemented in the control of a large-scale system proposed in Section V, where the obtained results are discussed. Finally, concluding remarks are drawn and further work is proposed in Section VI.

Notation: Column vectors are denoted by bold style, e.g., p. Matrices are denoted by bold upper case, e.g., A. Differently, scalars are denoted by non-bold style, e.g., $n$. The sets are denoted by calligraphic upper case, e.g., $\mathcal{S}$. The norm $\|\mathbf{x}\|_{\mathbf{Q}}^{2}$ is defined as $\|\mathbf{x}\|_{\mathbf{Q}}^{2}=\mathbf{x}^{\top} \mathbf{Q} \mathbf{x}$. The function $[\cdot]_{+}=\max (0, \cdot)$ is used to simplify the notation. The identity matrix of size $n \times n$ is denoted by $\mathbb{I}_{n}, \mathbb{1}_{n}$ is the column vector with $n$ unitary entries, i.e., $\mathbb{1}_{n}=\left[\begin{array}{lll}1 & \ldots & 1\end{array}\right]^{\top} \in \mathbb{R}^{n}$, similarly $\mathbf{0}_{n}$ is the column vector with $n$ null entries, i.e., $\mathbf{0}_{n}=\left[\begin{array}{lll}0 & \ldots & 0\end{array}\right]^{\top} \in \mathbb{R}^{n}$, in addition, $\mathbf{0}_{n \times l}$ is the matrix of null entries and dimension $n \times l$, and $\operatorname{diag}(\mathbf{x})$ is the diagonal matrix of the vector $\mathbf{x}$. Finally, real numbers are denoted by $\mathbb{R}$, and all the non-negative real numbers are denoted by $\mathbb{R}_{\geq 0}$. The non-negative and strictly positive integer numbers are denoted by $\mathbb{Z}_{\geq 0}$, and $\mathbb{Z}_{>0}$, respectively. Regarding the discrete time notation for the MPC controller, $\mathbf{x}_{k+j \mid k}$ denotes the prediction made at time $k$ of the vector $\mathbf{x}$ for time $k+j$, where $k, j \in \mathbb{Z}_{\geq 0}$, i.e., in the argument $k+j \mid k$, the first element $k+j$ indicates discrete time for prediction, whereas the second element $k$ indicates the actual discrete time. The use of continuous time denoted by $t$ is mostly omitted in order to simplify the notation.

Graphs: Graph-theoretical tools are used in order to describe possible interactions among strategies in the population-games context, and to describe the possible communication among different local control entities from the control perspective. The undirected graph is denoted by $\mathcal{G}=(\mathcal{S}, \mathcal{E}, \tilde{\mathbf{A}})$, where $\mathcal{S}=\{1, \ldots, n\}$ is the set of nodes (strategies in a population); $\mathcal{E} \subset\{(i, j): i, j \in \mathcal{S}\}$ is the set of links describing the possible communication sharing and/or interaction among agents selecting different strategies; and $\tilde{\mathbf{A}}=\left[\tilde{a}_{i j}\right]$ is an $n \times n$ adjacency matrix where $\tilde{a}_{i j}=1$ if $(i, j) \in \mathcal{E}$, and $\tilde{a}_{i j}=0$ otherwise. The set of neighbors of a node $i \in \mathcal{S}$ is given by $\mathcal{N}_{i}=\{j:(i, j) \in \mathcal{E}\}$.

\section{Population Games}

Consider a population composed of a large and finite number of agents. These agents select to belong to a strategy from the set of $n$ strategies given by $\mathcal{S}=\{1, \ldots, n\}$. The scalar $p_{i} \in \mathbb{R}_{\geq 0}$ corresponds to the portion of agents, which are selecting the strategy $i \in \mathcal{S}$. Therefore, the vector $\mathbf{p} \in \mathbb{R}_{\geq 0}^{n}$ represents the population state or the distribution of agents throughout the strategies. Different from the population dynamics without density dependence, the set of possible population states, denoted by $\Delta$, is given by the positive orthant, i.e., $\Delta=\left\{\mathbf{p} \in \mathbb{R}_{\geq 0}^{n}\right\}$. The size of the population is denoted by $m \in \mathbb{R}_{\geq 0}$, and given by $m=\sum_{i \in \mathcal{S}} p_{i}$, which may vary along the time since the population is a density-dependent process. Agents make the decisions to select among the different strategies pursuing to increment their reproduction chances in order to have more offspring. The mentioned incentives (reproduction chances) are represented by a fitness function denoted by $f_{i}(\mathbf{p})$, for all $i \in \mathcal{S}$, whose mapping is $f_{i}: \mathbb{R}_{\geq 0}^{n} \mapsto \mathbb{R}$. Function $f_{i}$ takes a population state and returns a benefit corresponding to strategy $i \in \mathcal{S}$. The vector of fitness functions for the entire population is denoted by $\mathbf{F}(\mathbf{p})$, whose mapping is given by $\mathbf{F}: \mathbb{R}_{\geq 0}^{n} \mapsto \mathbb{R}^{n}$. Function $\mathbf{F}$ takes a population state and returns a vector of benefits for the population.

It is assumed that agents are able to migrate from a strategy $i \in \mathcal{S}$ to a strategy $j \in \mathcal{S}$ following a revision protocol denoted by $\rho_{i j}(\mathbf{F}(\mathbf{p}), \mathbf{p})$, which defines how agents make decisions seeking to enhance their benefits.

Definition 1: The revision protocol function is given by a mapping $\rho: \mathbb{R}^{n} \times \mathbb{R}_{\geq 0}^{n} \mapsto \mathbb{R}_{\geq 0}^{n \times n}$ that describes the timing and the results of the agents decisions in the strategic interaction. Function $\rho$ takes values corresponding to the population state and its respective benefits, and returns a non-negative matrix representing decisions of agents about switching strategies [8].

The evolution process for the portion of agents within a strategic interaction is described by the general dynamics called the mean dynamics [8]. In [1], the distributed mean dynamics

$$
\dot{p}_{i}=\sum_{j \in \mathcal{N}_{i}} p_{j} \rho_{j i}(\mathbf{F}(\mathbf{p}), \mathbf{p})-p_{i} \sum_{j \in \mathcal{N}_{i}} \rho_{i j}(\mathbf{F}(\mathbf{p}), \mathbf{p}), \forall i \in \mathcal{S},
$$

are proposed for the deduction of distributed population dynamics. The framework of this work is given by fullpotential games. This class of games is formally defined next [8].

Definition 2: If there exists a continuously differentiable function $V(\mathbf{p})$, known as potential function, such that $\mathbf{F}(\mathbf{p})=\nabla V(\mathbf{p})$, for all $\mathbf{p} \in \Delta$, then $\mathbf{F}$ is a full-potential game.

\section{Density-dependent population games (DDPG)}

The density-dependent mean dynamics are obtained from the mean dynamics (1) including a function of reproduction 
rate denoted by $\delta_{i}$, for all $i \in \mathcal{S}$, i.e.,

$$
\dot{p}_{i}=\sum_{j \in \mathcal{N}_{i}} p_{j} \rho_{j i}(\mathbf{F}(\mathbf{p}), \mathbf{p})-p_{i} \sum_{j \in \mathcal{N}_{i}} \rho_{i j}(\mathbf{F}(\mathbf{p}), \mathbf{p})+\delta_{i}(\mathbf{F}(\mathbf{p})) \text {. }
$$

When $\delta_{i}>0$, then there is birth in the strategy $i \in \mathcal{S}$ since there are positive conditions promoting reproduction. On the other hand, if $\delta_{i}<0$, then there is death (interpretation of negative reproduction rates) in the strategy $i \in \mathcal{S}$ due to the fact that there are not ideal conditions for reproduction. In this regard, $\delta_{i}$ should be directly proportional to the fitness function $f_{i}$, i.e., successful agents (those with greater fitness functions) have more chances to have offspring [5].

Definition 3: The reproduction rate is a function with mapping $\delta: \mathbb{R}_{\geq 0}^{n} \mapsto \mathbb{R}^{n}$, and satisfying that reproduction rates decline as proportion of agents increases [5].

\section{DISTRIBUTED DENSITY-DEPENDENT REPLICATOR DYNAMICS}

In this work, the distributed density-dependent replicator dynamics (D3RD) are used. In order to deduce a version of the $\mathrm{D} 3 \mathrm{RD}$, the pairwise proportional imitation protocol is used, which is given by $\rho_{i j}(\mathbf{F}(\mathbf{p}), \mathbf{p})=p_{j}\left[f_{j}(\mathbf{p})-f_{i}(\mathbf{p})\right]_{+}$. Notice that the interpretation of the revision protocol dictates that an agent selecting a strategy $i \in \mathcal{S}$, comparing itself with an agent selecting strategy $j \in \mathcal{S}$, decides to move to $j \in \mathcal{S}$ only if the change represents an improvement over its reproductive chances, i.e., if $f_{j}(\mathbf{p})>f_{i}(\mathbf{p})$, and the switch rate is made in relation to the amount of agents $p_{j}$ selecting strategy $j \in \mathcal{S}$. Furthermore, the reproduction rate function is selected to be of the form $\delta_{i}(\mathbf{F}(\mathbf{p}))=\beta_{i} f_{i}(\mathbf{p})$, where $\beta_{i} \geq 0$ can be either constant or time varying, e.g., $\beta_{i}=1$, or $\beta_{i}=p_{i}$.

Notice that under the framework of stable games [8], $\mathbf{F}(\mathbf{p})$ is decreasing with respect to the amount of agents $\mathbf{p}$. The proposed reproduction rate function is suitable according to Definition 3 since $f_{i}$ declines as $p_{i}$ increases, then $\delta_{i}$ also declines as $p_{i}$ increases. Replacing $\rho_{i j}(\mathbf{F}(\mathbf{p}), \mathbf{p})$, and $\delta_{i}(\mathbf{F}(\mathbf{p})$ in (2) yields that for all $i \in \mathcal{S}$

$$
\dot{p}_{i}=p_{i}\left(f_{i}(\mathbf{p}) \sum_{j \in \mathcal{N}_{i}} p_{j}-\sum_{j \in \mathcal{N}_{i}} p_{j} f_{j}(\mathbf{p})\right)+\beta_{i} f_{i}(\mathbf{p}) .
$$

The equilibrium point of (3) is a Nash equilibrium denoted by $\mathbf{p}^{*}$, where $f_{i}\left(\mathbf{p}^{*}\right)=f_{j}\left(\mathbf{p}^{*}\right)$, for all $i, j \in \mathcal{S}$. Additionally, the reproduction rate makes to achieve a equilibrium such that $\mathbf{F}\left(\mathbf{p}^{*}\right)=\mathbf{0}_{n}{ }^{[1]}$. The interpretation of this situation from a biological perspective is that there is no agent with incentives to move among strategies, since it could not increase its reproduction chances. Likewise, there is an equilibrium between birth and death $\left(\delta_{i}=0\right.$, for all $\left.i \in \mathcal{S}\right)$, such that the population size remains constant. Furthermore, the D3RD (3) can be re-written in a compacted way as in [1], i.e.,

$$
\dot{\mathbf{p}}=\mathbf{L}^{(\mathbf{p})} \mathbf{F}(\mathbf{p})+\operatorname{diag}(\boldsymbol{\beta}) \mathbf{F}(\mathbf{p}),
$$

\footnotetext{
${ }^{1}$ The Nash equilibrium $\mathbf{p}^{*}$ implies that $\mathbf{F}\left(\mathbf{p}^{*}\right)=\mathbf{0}_{n}$ only for the population mass $m=\sum_{i \in \mathcal{S}} p_{i}^{*}$. In general, for a different population mass, the Nash equilibrium of the game $\mathbf{F}$ does not imply that $\mathbf{F}\left(\mathbf{p}^{*}\right)=\mathbf{0}_{n}$.
}

where $\boldsymbol{\beta} \in \mathbb{R}_{>0}^{n}$ is the vector of all the $\beta_{i}$ for all $i \in \mathcal{S}$, and $\mathbf{L}^{(\mathbf{p})}=\left[l_{i j}^{(\mathbf{p})}\right]$ is a matrix depending on the population state $\mathbf{p}$, whose entries are $l_{i j}^{(\mathbf{p})}=-a_{i j} p_{i} p_{j}$, if $i \neq j$, and $\sum_{k \in \mathcal{S}, \ell \neq i} a_{i \ell} p_{i} p_{\ell}$, if $i=j$. Once the D3RD have been formally introduced, it follows to make a stability analysis of the equilibrium point. Then, it is shown that the mentioned Nash equilibrium $\mathbf{p}^{*} \in \Delta$ is asymptotically stable under the D3RD with region of attraction given by the positive orthant as it is stated in Theorem 1.

Theorem 1: Let $\mathbf{F}$ be a full-potential game with strictly concave potential function $V(\mathbf{p})$, and let $\mathbf{p}^{*} \in \Delta$ be a Nash equilibrium for a corresponding population size $m \in \mathbb{R}_{\geq 0}$ such that $\mathbf{F}\left(\mathbf{p}^{*}\right)=\mathbf{0}_{n}$. If the strategy-interaction structure is given by a connected graph $\mathcal{G}$, then $\mathbf{p}^{*} \in \Delta$ is asymptotically stable under the D3RD (3).

Proof: It is considered the same Lyapunov candidate function as in [1], i.e., $E_{V}(\mathbf{p})=V\left(\mathbf{p}^{*}\right)-V(\mathbf{p})$, where $E_{V}\left(\mathbf{p}^{*}\right)=0$, and $E_{V}(\mathbf{p})>0$, for all $\mathbf{p} \neq \mathbf{p}^{*}$. It follows that $\dot{E}_{V}(\mathbf{p})=-(\nabla V(\mathbf{p}))^{\top} \dot{\mathbf{p}}$, which is the same as $\dot{E}_{V}(\mathbf{p})=$ $-\mathbf{F}(\mathbf{p})^{\top} \dot{\mathbf{p}}$. Now, replacing $\dot{\mathbf{p}}$ from (4), it is obtained that

$$
\dot{E}_{V}(\mathbf{p})=-\mathbf{F}(\mathbf{p})^{\top} \mathbf{L}^{(\mathbf{p})} \mathbf{F}(\mathbf{p})-\mathbf{F}(\mathbf{p})^{\top} \operatorname{diag}(\boldsymbol{\beta}) \mathbf{F}(\mathbf{p}) .
$$

Notice that $\mathbf{L}^{(\mathbf{p})}$ corresponds to the Laplacian of a graph $\mathcal{G}^{(\mathbf{p})}=\left(\mathcal{V}, \mathcal{E}, \tilde{\mathbf{A}}^{(\mathbf{p})}\right)$, where $\tilde{\mathbf{A}}^{(\mathbf{p})}=\left[\tilde{a}_{i j}^{(\mathbf{p})}\right]$ is the adjacency matrix with entries given by $\tilde{a}_{i j}^{(\mathbf{p})}=\tilde{a}_{i j} p_{i} p_{j}$. The entries of the adjacency matrix are non-negative due to the fact that $\mathbf{p} \in \Delta$, which is the positive orthant. Therefore $\mathbf{L}^{(\mathbf{p})} \geq 0$. Additionally, the diagonal matrix is $\operatorname{diag}(\boldsymbol{\beta}) \geq 0$ due to the fact that $\beta_{i} \geq 0$ for all $i \in \mathcal{S}$. Finally, it is concluded that $\dot{E}_{V}(\mathbf{p}) \leq 0$. Moreover, the equality $\dot{E}_{V}(\mathbf{p})=0$ holds when $\mathbf{F}(\mathbf{p})=\mathbf{0}_{n}$, and therefore $\mathbf{p}^{*}$ is asymptotically stable under the D3RD (3) with region of attraction $\Delta$.

Corollary 1: The asymptotic stability of $\mathbf{p}^{*} \in \Delta$ under the D3RD (3) stated in Theorem 1 holds for connected timevarying graphs $\mathcal{G}(t)=(\mathcal{V}, \mathcal{E}(t), \tilde{\mathbf{A}}(t))$. This statement can be seen from Theorem 1 since the Lyapunov function is common for all possible connected graph topologies.

\section{A. Solving constrained optimization problems with DDPG}

Consider a quadratic programming (QP) optimization problem of the form

$$
\max _{\mathbf{y}} f(\mathbf{y}), \text { s. t. } \mathbf{E y} \leq \mathbf{e}, \quad \mathbf{G y}=\mathbf{g}, \text { and } \mathbf{y} \in \mathbb{R}_{\geq 0}^{v},
$$

where $f: \mathbb{R}_{\geq 0}^{v} \mapsto \mathbb{R}$ is concave, and continuously differentiable. Moreover, $\mathbf{E} \in \mathbb{R}^{q \times v}$, and $\mathbf{e} \in \mathbb{R}^{q}$ construct the $q$ inequality constraints, and $\mathbf{G} \in \mathbb{R}^{r \times v}$, and $\mathbf{g} \in \mathbb{R}^{r}$ construct the $r$ equality constraints. Inequality constraints can be transformed into equality constraints by adding nonnegative slack variables denoted by $\mathbf{s} \in \mathbb{R}_{>0}^{q}$. To this end, consider the vector variable $\boldsymbol{\xi}=\left[\begin{array}{ll}\mathbf{y}^{\top} & \mathbf{s}^{\top}\end{array}\right]^{\top} \in \mathbb{R}^{p}$, where $p=v+q$, then the $\mathrm{QP}$ optimization problem (6) is reformulated as follows:

$$
\max _{\boldsymbol{\xi}} f(\boldsymbol{\xi}), \text { s. t. } \mathbf{H} \boldsymbol{\xi}=\mathbf{h}, \text { and } \boldsymbol{\xi} \in \mathbb{R}_{\geq 0}^{p},
$$

where $f: \mathbb{R}_{>0}^{p} \mapsto \mathbb{R}$ is concave and continuously differentiable, and $\mathbf{H} \in \mathbb{R}^{w \times p}$, and $\mathbf{h} \in \mathbb{R}^{w}$ construct the $w$ equality 
constraints, where $w=q+r$. Now, omitting the positiveness constraints (7) (due to the fact that population dynamics only evolve in the positive orthant $\Delta$ ), the Lagrangian function $l: \mathbb{R}^{p} \times \mathbb{R}^{w} \mapsto \mathbb{R}$ is $l(\boldsymbol{\xi}, \boldsymbol{\mu})=f(\boldsymbol{\xi})+\boldsymbol{\mu}^{\top}(\mathbf{H} \boldsymbol{\xi}-\mathbf{h})$, where $\boldsymbol{\mu} \in \mathbb{R}^{w}$ corresponds to the Lagrange multipliers associated to the $w$ equality constraints of (7). Moreover, $\nabla_{\boldsymbol{\xi}} l(\boldsymbol{\xi}, \boldsymbol{\mu})=\nabla f(\boldsymbol{\xi})+\mathbf{H}^{\top} \boldsymbol{\mu}$, and $-\nabla_{\boldsymbol{\mu}} l(\boldsymbol{\xi}, \boldsymbol{\mu})=-\mathbf{H} \boldsymbol{\xi}+\mathbf{h}$.

The Lagrange condition is used to find the possible extreme points $\boldsymbol{\xi}^{*} \in \mathbb{R}^{p}$ of the function $f(\boldsymbol{\xi})$ (maximum of the function $f$ ) subject to constraints (7), in which $\left[\nabla_{\boldsymbol{\xi}} l\left(\boldsymbol{\xi}^{*}, \boldsymbol{\mu}^{*}\right)^{\top}-\nabla_{\boldsymbol{\mu}} l\left(\boldsymbol{\xi}^{*}, \boldsymbol{\mu}^{*}\right)^{\top}\right]^{\top}=\mathbf{0}_{p+w}$. Now, let $\mathbf{p}=\left[\begin{array}{ll}\boldsymbol{\xi}^{\top} & \boldsymbol{\mu}^{\top}\end{array}\right]^{\top} \in \mathbb{R}^{n}$ be the vector representing the amount of agents in a strategic interaction with $\mathcal{S}=\{1, \ldots, n\}$, where $n=p+w$. Besides, let

$$
\mathbf{F}(\mathbf{p})=\left[\nabla_{\boldsymbol{\xi}} l(\boldsymbol{\xi}, \boldsymbol{\mu})^{\top}-\nabla_{\boldsymbol{\mu}} l(\boldsymbol{\xi}, \boldsymbol{\mu})^{\top}\right]^{\top}
$$

be the fitness functions corresponding to all the strategies $\mathcal{S}$. The population game (8) can be seen as two different potential games $\mathbf{F}_{\boldsymbol{\xi}}=\nabla_{\boldsymbol{\xi}} l(\boldsymbol{\xi}, \boldsymbol{\mu})$, and $\mathbf{F}_{\boldsymbol{\mu}}=-\nabla_{\boldsymbol{\mu}} l(\boldsymbol{\xi}, \boldsymbol{\mu})$, whose potential functions are $l(\boldsymbol{\xi}, \boldsymbol{\mu})$, and $-l(\boldsymbol{\xi}, \boldsymbol{\mu})$, respectively. Therefore, notice that the Hessian of the potential functions is $\nabla_{\xi}^{2} l(\boldsymbol{\xi}, \boldsymbol{\mu})=\nabla^{2} f(\boldsymbol{\xi})$, and $\nabla_{\boldsymbol{\mu}}^{2}(-l(\boldsymbol{\xi}, \boldsymbol{\mu}))=\mathbf{0}$. Therefore, $\mathbf{F}_{\xi}$, and $\mathbf{F}_{\mu}$ are stable games [8]. Finally, according to Theorem 1 and Corollary 1, the optimization problem (6) can be solved in a distributed way by using the D3RD (3), and under time-varying graphs $\mathcal{G}(t)^{[2]}$.

\section{DistRIBUTED MPC BASED ON DDPG WITH TIME-VARYING COMMUNICATION NETWORK}

Consider a state-space discrete-time system with a sampling time $\Delta t$ and represented by the following model:

$$
\mathbf{x}_{k+1}=\mathbf{A} \mathbf{x}_{k}+\mathbf{B} \mathbf{u}_{k}+\mathbf{B}_{d} \mathbf{d}_{k},
$$

where $\mathbf{x} \in \mathbb{R}^{n_{x}}$ is the system state vector, $\mathbf{u} \in \mathbb{R}^{n_{u}}$ is the vector of control inputs, $\mathbf{d} \in \mathbb{R}^{n_{d}}$ denotes the vector of disturbances that affect the system, and the state-space matrices are given by $\mathbf{A} \in \mathbb{R}^{n_{x} \times n_{x}}, \mathbf{B} \in \mathbb{R}^{n_{x} \times n_{u}}$, and $\mathbf{B}_{d} \in \mathbb{R}^{n_{x} \times n_{d}}$. The states and control inputs are subject to physical and operational constraints, which define feasible sets denoted by $\mathcal{X} \triangleq\left\{\mathrm{x} \in \mathbb{R}^{n_{x}}: \mathrm{x}^{\min } \leq \mathrm{x} \leq \mathrm{x}^{\max }\right\}$, and $\mathcal{U} \triangleq\left\{\mathbf{u} \in \mathbb{R}^{n_{u}}: \mathbf{u}^{\min } \leq \mathbf{u} \leq \mathbf{u}^{\max }\right\}$, where vectors $\mathrm{x}^{\min }$ and $\mathrm{x}^{\max }$ correspond to the lower and upper limits for the system states, respectively. Similarly, vectors $\mathbf{u}^{\text {min }}$ and $\mathbf{u}^{\max }$ denote the lower and upper limits for the control inputs, respectively. The control sequence for a fixedtime prediction horizon, represented by $H_{p} \in \mathbb{Z}_{>0}$ at the instant time $k \in \mathbb{Z}_{\geq 0}$, is denoted by $\hat{\mathbf{u}}_{k}$. When the control input sequence $\hat{\mathbf{u}}_{k}$ is applied to the system (9) with initial state $\mathbf{x}_{k \mid k} \triangleq \mathbf{x}_{k}$, a system states sequence $\hat{\mathbf{x}}_{k}$ is generated, i.e., $\hat{\mathbf{u}}_{k} \triangleq\left\{\mathbf{u}_{k \mid k}, \mathbf{u}_{k+1 \mid k}, \ldots, \mathbf{u}_{k+H_{p}-1 \mid k}\right\}$, $\hat{\mathbf{x}}_{k} \triangleq\left\{\mathbf{x}_{k+1 \mid k}, \mathbf{x}_{k+2 \mid k}, \ldots, \mathbf{x}_{k+H_{p} \mid k}\right\}$. Finally, the disturbances time-varying sequence along the horizon $H_{p}$ is denoted by $\hat{\mathbf{d}}(k)$, i.e., $\hat{\mathbf{d}}_{k} \triangleq\left\{\mathbf{d}_{k \mid k}, \mathbf{d}_{k+1 \mid k}, \ldots, \mathbf{d}_{k+H_{p}-1 \mid k}\right\}$.

\footnotetext{
${ }^{2}$ Even though population dynamics only admit values within the positive orthant $\Delta$, an optimization problem allowing negative values can also been solved by using the D3RD. This is made by applying a change of variables in the fitness functions.
}

The discrete model (9) is controlled with an MPC law whose optimization problem, in terms of the slew rate $\Delta \mathbf{u}_{k+j}=\mathbf{u}_{k+j}-\mathbf{u}_{k+j-1}$, for all $j \in\left[1, H_{p}\right] \cap \mathbb{Z}_{>0}$, is as follows:

$$
\begin{aligned}
\min _{\hat{\mathbf{u}}} J(\mathbf{x}, \mathbf{u}) & =\sum_{j=1}^{H_{p}}\left\{\left\|\mathbf{x}_{k+j \mid k}-\mathbf{x}_{r}\right\|_{\tilde{\mathbf{Q}}}+\left\|\Delta \mathbf{u}_{k+j \mid k}\right\|_{\tilde{\mathbf{R}}}\right\}, \\
\text { s.t. } \mathbf{x}_{k+j+1 \mid k} & =\mathbf{A} \mathbf{x}_{k+j \mid k}+\mathbf{B} \mathbf{u}_{k+j \mid k}+\mathbf{B}_{d} \mathbf{d}_{k+j \mid k}, \\
\mathbf{u}_{k+j \mid k} & \in \mathcal{U}, \\
\mathbf{x}_{k+j \mid k} & \in \mathcal{X} \\
\mathbf{0}_{r} & =\mathbf{E}_{u} \mathbf{u}_{k+j \mid k}+\mathbf{E}_{d} \mathbf{d}_{k+j \mid k}
\end{aligned}
$$

where (10b), (10c), and (10e) with $j \in\left[0, H_{p}-1\right] \cap \mathbb{Z}_{\geq 0}$; and (10d) with $j \in\left[1, H_{p}\right] \cap \mathbb{Z}_{>0}$. Besides, $\mathbf{x}_{r} \in \mathbb{R}^{n_{x}}$ is a constant desired set-point for the system states $\mathbf{x} \in \mathbb{R}^{n_{x}}$. On the other hand, $\mathbf{E}_{u} \in \mathbb{R}^{r \times n_{u}}$, and $\mathbf{E}_{d} \in \mathbb{R}^{r \times n_{d}}$ construct the $r$ equality constraints in (10e). The matrices $\tilde{\mathbf{Q}} \in \mathbb{R}^{n_{x} \times n_{x}}$ and $\tilde{\mathbf{R}} \in \mathbb{R}^{n_{u} \times n_{u}}$ are weights assigning a prioritization for the control objectives related to the error and to energy slew rate, respectively. Assuming that the optimization problem (10) is feasible, an optimal sequence is computed, and following the MPC philosophy, a new optimization problem is formulated for the next iteration.

Then, some re-formulations over the cost function (10a) and constraints (10b)-(10e) are presented. These modifications are necessary in order to show the densitydependent population-games approach as an alternative tool for distributed MPC design. The optimization problem behind the MPC controller in (10) can be conveniently re-formulated with a cost function given by $J=\left(\mathbf{X}(k)-\mathbf{X}_{r}\right)^{\top} \mathbf{Q}\left(\mathbf{X}(k)-\mathbf{X}_{r}\right)+\Delta \mathbf{U}(k)^{\top} \mathbf{R} \Delta \mathbf{U}(k)$, where weighting matrices are $\mathbf{Q}=\operatorname{diag}\left(\left[\begin{array}{lll}\tilde{\mathbf{Q}} & \ldots & \tilde{\mathbf{Q}}\end{array}\right]\right)$ and $\mathbf{R}=\operatorname{diag}\left(\left[\begin{array}{lll}\tilde{\mathbf{R}} & \ldots & \tilde{\mathbf{R}}\end{array}\right]\right)$. The reference vector along $H_{p}$ is $\mathbf{X}_{r}=\left[\begin{array}{llll}\mathbf{x}_{r}^{\top} & \mathbf{x}_{r}^{\top} & \ldots & \mathbf{x}_{r}^{\top}\end{array}\right]^{\top}$, and vectors $\mathbf{X}_{k}$ and $\mathbf{U}_{k}$ are $\mathbf{X}_{k}=\left[\begin{array}{llll}\mathbf{x}_{k+1 \mid k}^{\top} & \mathbf{x}_{k+2 \mid k}^{\top} & \cdots & \mathbf{x}_{k+H_{p} \mid k}^{\top}\end{array}\right]^{\top}$, and $\mathbf{U}_{k}=\left[\begin{array}{llll}\mathbf{u}_{k \mid k}^{\top} & \mathbf{u}_{k+1 \mid k}^{\top} & \cdots & \mathbf{u}_{k+H_{p}-1 \mid k}^{\top}\end{array}\right]^{\top}$. Moreover, applying the appropriate transformations, the optimization problem can be re-written as a QP problem as follows:

$$
\min _{\Delta \mathbf{U}_{k}} \Delta \mathbf{U}_{k}^{\top} \boldsymbol{\Phi} \Delta \mathbf{U}_{k}+\phi_{k}^{\top} \Delta \mathbf{U}_{k}
$$

with constraints of the form as in (6). Moreover, for the optimization problem (11) the vectors $\mathbf{e}_{k}$ and $\mathbf{g}_{k}$, which construct the inequality and equality constraints, vary every iteration $k$. Furthermore, by adding non-negative slack variables $\mathbf{s} \in \mathbb{R}_{\geq 0}^{q}$, the cost function (11) is re-written, and the constraints can be compacted, i.e.,

$$
\begin{aligned}
& \min _{\boldsymbol{\xi}} \boldsymbol{\xi}_{k}^{\top} \underbrace{\left[\begin{array}{cc}
\boldsymbol{\Phi} & \mathbf{0}_{n_{u} \times q} \\
\mathbf{0}_{q \times n_{u}} & \mathbf{0}_{q \times q}
\end{array}\right]}_{\mathbf{\Psi}} \boldsymbol{\xi}_{k}+\underbrace{\left[\begin{array}{ll}
\boldsymbol{\phi}_{k}^{\top} & \mathbf{0}_{q}^{\top}
\end{array}\right]}_{\boldsymbol{\psi}} \boldsymbol{\xi}_{k}, \\
& \text { s. t. } \underbrace{\left[\begin{array}{cc}
\mathbf{E} & \mathbb{I}_{q} \\
\mathbf{G} & \mathbf{0}_{r \times q}
\end{array}\right]}_{\mathbf{H}} \boldsymbol{\xi}_{k}=\underbrace{\left[\begin{array}{c}
\mathbf{e}_{k} \\
\mathbf{g}_{k}
\end{array}\right]}_{\mathbf{h}},
\end{aligned}
$$

with $\boldsymbol{\xi}_{k}=\left[\begin{array}{ll}\Delta \mathbf{U}_{k}^{\top} & \mathbf{s}^{\top}\end{array}\right]^{\top}$. Having added the slack variables, the optimization problem behind the MPC is formulated of 
the form (7), and it can be solved in a distributed manner by using the D3RD (3) as explained in Section III.

Regarding the information dependence, it is mainly given by the fitness-functions coupling. In order to determine the communication structure for the D3RD, the fitness functions (8) are expressed in the form

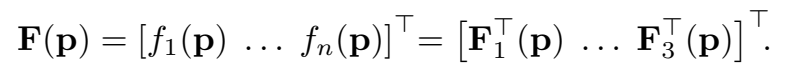

First block $\mathbf{F}_{1}(\mathbf{p})=\left[\begin{array}{llll}\nabla_{p_{1}} l(\mathbf{p}) & \ldots & \nabla_{p_{n_{u}}} l(\mathbf{p})\end{array}\right]^{\top}$ of the vector $\mathbf{F}(\mathbf{p})$ in (12) corresponds to the $n_{u}$ variables $\Delta u$, the second block $\mathbf{F}_{2}(\mathbf{p})=-\left[\nabla_{p_{\left(n_{u}+1\right)}} l(\mathbf{p}) \ldots \nabla_{p_{\left(n_{u}+q\right)}} l(\mathbf{p})\right]^{\top}$ corresponds to the $q$ inequality constraints, and the third block $\mathbf{F}_{3}(\mathbf{p})=-\left[\begin{array}{llll}\nabla_{p_{\left(n_{u}+q+1\right)}} l(\mathbf{p}) & \ldots & \nabla_{p_{\left(n_{u}+q+r\right)}} l(\mathbf{p})\end{array}\right]^{\top}$ corresponds to the $r$ equality constraints.

In order to check from which strategies it is necessary to get information, the Hessian matrix of the Lagrangian function $\boldsymbol{\Theta}=\left[\boldsymbol{\theta}_{i j}\right]=\nabla^{2} l(\mathbf{p})$ is computed, i.e., $\boldsymbol{\theta}_{i j}=\nabla_{p_{j}} f_{i}(\mathbf{p})$. Then, the biggest required communication matrix denoted by $\tilde{\boldsymbol{\Theta}}=\left[\tilde{\boldsymbol{\theta}}_{i j}\right]$, which corresponds to the existing coupling among the portion of agents at each strategy, is given by $\tilde{\boldsymbol{\theta}}_{i j}=1$, if $\boldsymbol{\theta}_{i j} \neq 0$, and $\tilde{\boldsymbol{\theta}}_{i j}=0$, otherwise.

For the time-invariant graph $\mathcal{G}$ scenario, the adjacency matrix is given by the biggest required communication matrix, i.e., $\tilde{\mathbf{A}}=\tilde{\mathbf{\Theta}}$. Nevertheless, conditions over the adjacency matrix can be added in order to use less communication links when conveniently.
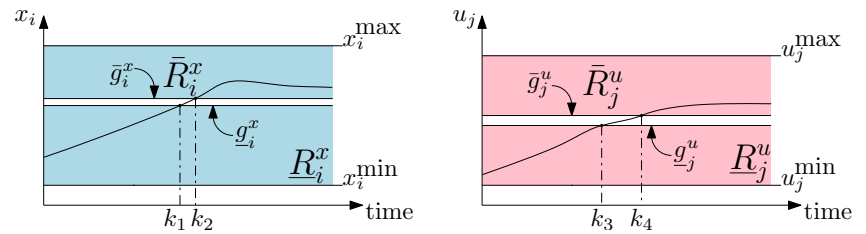

Fig. 1. Different regions for the consideration of inequality constraints. Additionally $x_{i}^{\min } \mathbb{1}_{2}^{\top} \leq\left[\begin{array}{ll}\bar{g}_{i}^{x} & \underline{g}_{i}^{x}\end{array}\right] \leq x_{i}^{\max } \mathbb{1}_{2}^{\top}$ for all $i=1, \ldots, n_{x}$, and $u_{j}^{\min } \mathbb{1}_{2}^{\top} \leq\left[\begin{array}{ll}\bar{g}_{j}^{u} & \underline{g}_{j}^{u}\end{array}\right] \leq u_{j}^{\max } \mathbb{1}_{2}^{T^{i}}$ for all $j=1, \ldots, n_{u}$.

In this paper, it is proposed to have a communication graph topology depending on the necessary active constraints. This is made since, under some system state conditions, it is not necessary to consider the whole set of constraints. In this regard, some of them can be properly neglected reducing both the size of the communication network and the computational burden. Figure 1 shows different possible regions for states and control inputs established by parameters $\bar{g}_{i}^{x}, \underline{g}_{i}^{x}, \bar{g}_{i}^{u}$, and $\underline{g}_{i}^{u}$, which are constant values determined at a design stage.

Regions describe non-safe sectors in the feasible set, or sectors near limits of a constraint. Figure 1 presents two examples, i.e., for a time $<k_{1}$, the constraint $x_{i} \geq x_{i}^{\min }$ is active whereas the constraints $x_{i} \leq x_{i}^{\max }$ is neglected. Then, for the time $>k_{2}$, the constraint $x_{i} \geq x_{i}^{\min }$ is neglected whereas the constraints $x_{i} \leq x_{i}^{\max }$ is considered. Similarly, Figure 1 also shows the scenario of control input $u_{j}$ with the respective time instants $k_{3}$, and $k_{4}$. The regions, shown in Figure 1, are defined as follows:

- Upper region for states: $\bar{R}_{i}^{x}=\left\{x_{i}: \bar{g}_{i}^{x} \leq x_{i} \leq x_{i}^{\max }\right\}$,

- Lower region for states: $\underline{R}_{i}^{x}=\left\{x_{i}: x_{i}^{\min } \leq x_{i} \leq \underline{g}_{i}^{x}\right\}$,

- Upper region for inputs: $\bar{R}_{j}^{u}=\left\{u_{j}: \bar{g}_{j}^{u} \leq u_{j} \leq u_{j}^{\frac{2}{\max }}\right\}$,
- Lower region for inputs: $\underline{R}_{j}^{u}=\left\{u_{j}: u_{j}^{\min } \leq u_{j} \leq \underline{g}_{j}^{u}\right\}$, for all $i=1, \ldots, n_{x}$, and $j=1, \ldots, n_{u}$. Besides, binary variables $\bar{\gamma}_{i, k}^{x}, \underline{\gamma}_{i, k}^{x}$, which indicate whether or not the current state $x_{i, k}$ belongs to a region, are defined as follows:

$\bar{\gamma}_{i, k}^{x}=\left\{\begin{array}{c}1, \text { if } x_{i, k} \in \bar{R}_{i}^{x} \\ 0, \text { otherwise }\end{array}, \quad \underline{\gamma}_{i, k}^{x}=\left\{\begin{array}{c}1, \text { if } x_{i, k} \in \underline{R}_{i}^{x} \\ 0, \text { otherwise }\end{array}\right.\right.$

where $i=1, \ldots, n_{x}$. The parameters $\bar{\gamma}_{j, k}^{u}$, and $\underline{\gamma}_{j, k}^{u}$, indicating whether or not the current control input $u_{i, k}$ is within a region, are stated similarly for $j=1, \ldots, n_{u}$. These binary variables lead to a vector that determines the active and non-active constraints for states and control inputs at each time instant, i.e., $\boldsymbol{\Gamma}_{k}^{u}=\left[\bar{\gamma}_{1, k}^{u}, \ldots, \bar{\gamma}_{n_{u}, k}^{u}, \underline{\gamma}_{1, k}^{u}, \ldots, \underline{\gamma}_{n_{u}, k}^{u}\right]^{\top}$, $\boldsymbol{\Gamma}_{k}^{x}=\left[\bar{\gamma}_{1, k}^{x}, \ldots, \bar{\gamma}_{n_{x}, k}^{x}, \underline{\gamma}_{1, k}^{x}, \ldots, \underline{\gamma}_{n_{x}, k}^{u}\right]^{\top}$. Then, let $\boldsymbol{\Gamma}_{k}$ be the diagonal matrix of the active constraints at instant time $k \in \mathbb{Z}_{\geq 0}$, i.e., $\tilde{\boldsymbol{\Gamma}}_{k}=\operatorname{diag}\left(\left[\begin{array}{llll}\mathbb{1}_{n_{u}}^{\top} & \boldsymbol{\Gamma}_{k}^{u \top} & \boldsymbol{\Gamma}_{k}^{x \top} & \mathbb{1}_{r}^{\top}\end{array}\right]\right)$.

Finally, these conditions over the active constraints lead to a time-varying graph $\tilde{\mathbf{A}}(t)$ that varies its topology at every $\Delta t$, given by $\tilde{\mathbf{A}}(k \Delta t)=\tilde{\boldsymbol{\Gamma}}_{k} \tilde{\boldsymbol{\Theta}} \tilde{\boldsymbol{\Gamma}}_{k}$, and the topology $\tilde{\mathbf{A}}(k \Delta t)$ is maintained during a time $\Delta t$, i.e., $\tilde{\mathbf{A}}(t)=\tilde{\mathbf{A}}(k \Delta t)$, for all $k \Delta t \leq t<(k+1) \Delta t$.

\section{CASE STUdy AND SIMUlation RESUlts}

The portion of the Barcelona Drinking Water Network (BDWN) presented in [2] is considered. It is a large-scale system composed of $n_{x}=17$ tanks, $n_{u}=61$ control inputs (valves and pumps), nine drinking water sources, and $n_{d}=$ 25 water demands as reported in [6]. State vector $\mathbf{x} \in \mathbb{R}^{n_{x}}$, the vector of control inputs $\mathbf{u} \in \mathbb{R}^{n_{u}}$, and the vector of disturbances $\mathbf{d} \in \mathbb{R}^{n_{d}}$ are associated to volumes in tanks, manipulated flows, and water-demanded flows, respectively. The corresponding discrete-time model is the one presented in (9) and its sampling time is $\Delta t=1$ hour. The description of the static mass balance at junction nodes in the network is given by (10e).

The control objectives are to meet a reference, which determines a safety volume to satisfy the time-varying demand, and to minimize the variation of the flows in order to avoid abrupt changes, which might cause damage in the network (10a). For the simulation results, the following parameters are selected: $\mathbf{x}_{r}=0.6 \mathbf{x}^{\max }, \tilde{\mathbf{Q}}=\mathbb{I}_{n_{x}}$, $\tilde{\mathbf{R}}=1000 \mathbb{I}_{n_{u}}$. The regions to determine the activeness of constraints are computed by using the following parameters: $\bar{g}_{i}^{x}=\underline{g}_{i}^{x}=0.6 x_{i}^{\max }$, for all $i=1, \ldots, n_{x}$; and $\bar{g}_{j}^{u}=0.65 \bar{u}_{j}^{\max }$, and $\underline{g}_{j}^{u}=0.35 u_{j}^{\max }$, for all $j=1, \ldots, n_{u}$.

Figure 2(a) presents the communication topology required to solve the optimization problem by using the $\mathrm{D} 3 \mathrm{RD}$ when all the inequality constraints are active, i.e., $\tilde{\mathbf{A}}(t)=\tilde{\boldsymbol{\Theta}}$. Figure 2(b) corresponds to the graph when adopting time-varying graphs and the proposed distributed density-dependent population-dynamics-based MPC controller. Graph in Figure 2(b) has a reduction of $36.16 \%$ of the communication links, which is produced due to the fact that, at $k=77$, a total of 58 inequality constraints are nonactive. Figures 3(a)-3(b) show the evolution of some states achieving the imposed reference, also reflecting a proper 


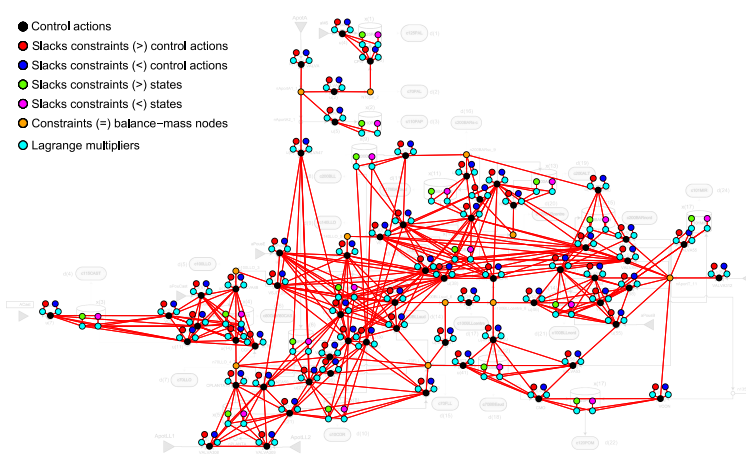

(a)

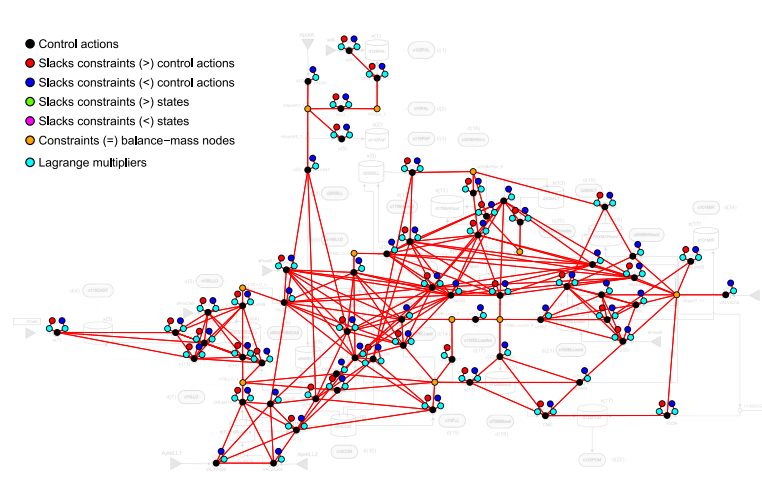

(b)

Fig. 2. Communication topologies for the distributed density-dependent population-games-based MPC. (a) communication topology for the distributed MPC based on D3RD, i.e., $\tilde{\mathbf{A}}=\tilde{\boldsymbol{\Theta}}$. The number of links in this graph for this case study is 614 ; and (b) time-varying communication topology for the distributed MPC based on D3RD at time instant $k=77$, i.e., $\tilde{\mathbf{A}}(77 \Delta t)=\tilde{\boldsymbol{\Gamma}}(77) \tilde{\Theta} \tilde{\boldsymbol{\Gamma}}(77)$. The number of links in this graph for this case study is 392 .
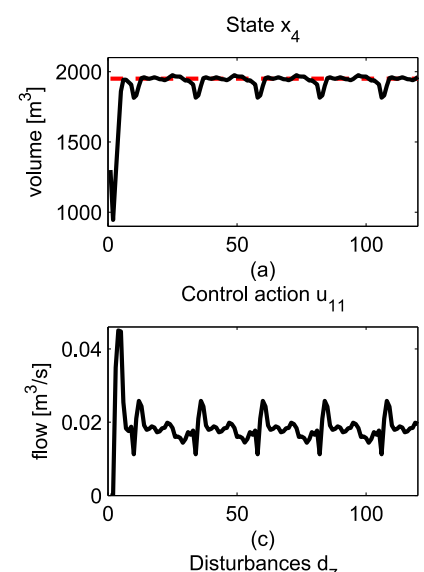

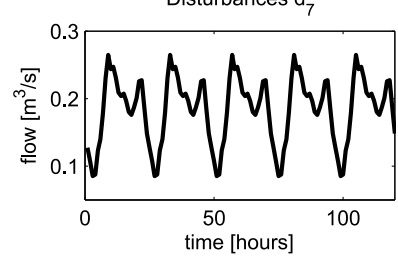

(e)
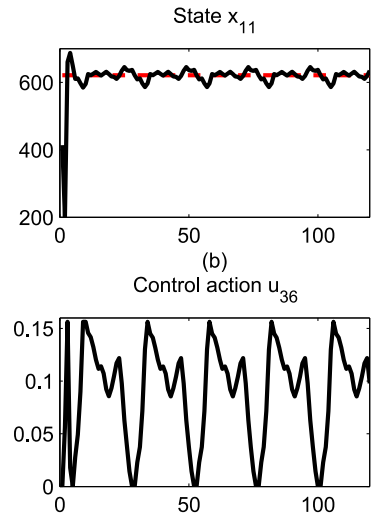

(d)

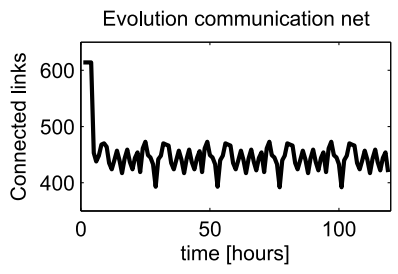

(f)
Fig. 3. The evolution of states $x_{4}$ and $x_{11}$ are presented in (a) and (b). The evolution of control inputs $u_{11}$ and $u_{36}$ are presented in (c) and (d). An example of the demand profiles is presented in (e) corresponding to $d_{7}$. Finally, the number of connected communication links along the time is presented in (f). The minimum achieved number of links is 392 corresponding to Figure 2(b).

performance of the proposed distributed density-dependent population-dynamics-based MPC achieving the references. On the other hand, Figures 3(c)-3(d) present the behavior of some control signals. It can be seen that these control inputs oscillate in order to satisfy the constraints imposed by the evolution of the demanded flows. That is why, these control inputs have the same periodicity as the disturbances (period of 24 hours). Although all the demands have different magnitudes and mean values, they have the same daily periodicity as the disturbance $d_{7}$ presented in Figure 3(e). Finally, Figure 3(f) shows the evolution of the number of connected links in the communication network along the time. It can be seen that, at the beginning, it is needed to have the communication graph corresponding to the biggest required communication matrix, i.e., $\tilde{\mathbf{A}}(t)=\tilde{\mathbf{\Theta}}$. Moreover, it can be seen a periodic behavior of the number of required links in the communication network, is daily (period of 24 hours) as the disturbances.

\section{CONCLUSIONS AND FURTHER WORK}

A general methodology to generate distributed densitydependent population dynamics has been presented by considering a reproduction rate in the distributed mean dynamics. Furthermore, it has been shown the relationship between the equilibrium point of density games with the optimal point in a constrained optimization problem by selecting the description of benefits throughout the strategies using the Lagrangian function. Besides, the asymptotic stability of the equilibrium point under the D3RD has been formally proven for constant and time-varying strategy-constraint structures. Then, this class of dynamics has been applied in the design of a distributed MPC controller under a time-varying communication network. Simulation results have shown a reduction in the number of links of the communication network over a $36 \%$ with respect to the total number of links required by the distributed MPC under a fixed communication network. As further work, it is highlighted that other population dynamics such as the Smith or projection, among others, may be deduced for their distributed density-dependent version by following the same procedure presented in this paper.

\section{REFERENCES}

[1] J Barreiro-Gomez, G Obando, and N Quijano. Distributed population dynamics: Optimization and control applications. IEEE Transactions on Systems, Man, and Cybernetics: Systems, 99:1-11, 2016.

[2] J. Barreiro-Gomez, N. Quijano, and C. Ocampo-Martinez. Constrained distributed optimization: A population dynamics approach. Automatica, 69:101-116, 2016.

[3] R. Cressman and V. Krrivan. Migration dynamics for the ideal free distribution. The American Naturalist, 168(3):384-397, 2006.

[4] R. Negenborn and J.M. Maestre. Distributed model predictive control: An overview and roadmap of future research opportunities. IEEE Control Systems, 34(4):87-97, 2014.

[5] S. Novak, K. Chatterjee, and M.A. Nowak. Density games. Journal of Theoretical Biology, 334(2013):26-34, 2013.

[6] C. Ocampo-Martinez, V. Puig, G. Cembrano, and J. Quevedo. Application of predictive control strategies to the management of complex networks in the urban water cycle. IEEE Control Systems, 33(1):15-41, 2013.

[7] E. Ramirez-Llanos and N. Quijano. A population dynamics approach for the water distribution problem. International Journal of Control, 83(9):1947-1964, 2010.

[8] W. H. Sandholm. Population games and evolutionary dynamics. Cambridge, Mass. MIT Press, 2010. 